\title{
Quimioterapia de rescate basada en brentuximab en linfoma Hodgkin y linfomas no Hodgkin de células T
}

\author{
- Leonardo Enciso, Bonell Patiño, Mónica Arévalo, Martha Díaz, Humberto Martínez, Paola Spirko, Diana Otero
}

Correo electrónico: humbertomartinez48@hotmail.com

Introducción: describir los resultados del tratamiento con brentuximab vedotin (BV) en el tratamiento de rescate de linfoma Hodgkin (LH), linfomas de células T (LCTP) y linfoma de la zona gris (LZG), y como estrategia de consolidación luego de trasplante autólogo (TA).

Métodos: estudio descriptivo tipo serie de casos. Pacientes mayores de 18 años con diagnóstico de LCTP de cualquier subtipo y LH con al menos un ciclo de tratamiento con BV. Todos los análisis fueron realizados en los paquetes estadísticos R (versión 3.4.3) y Stata 15.

Resultados: un total de 196 ciclos de brentuximab se administró en 27 pacientes. El 70,37\% eran hombres. La mediana de edad fue 30,8 años. El 70,37\% tenía diagnóstico de LH. El tiempo desde el diagnóstico hasta el inicio del brentuximab fue de 3,99 años, con un promedio de 3,16 líneas previas. Nueve pacientes recibieron brentuximab como consolidación pos-TA. El BV se usó como monoterapia en 21 pacientes $(77,8 \%)$ y en 6 en combinación con bendamustine, estos últimos todos con LH. El $14,81 \%$ requirió al menos una hospitalización por complicaciones. La respuesta global fue del $41,17 \%$. La mediana de supervivencia libre de progresión fue de 2,38 años (IC95\% 1,24 - NR). La supervivencia global no fue alcanzada con una mediana de seguimiento de 30 meses. El 33,3\% precisó un nuevo tratamiento.

Conclusión: la terapia con BV es segura, con buenas tasas de respuesta, incluso con múltiples líneas de tratamiento previas. Se observan mejores desenlaces en los pacientes que son llevados a TA.

\section{Crisis blástica mielomastocítica en leucemia mieloide crónica resistente a inhibidores de tirosina quinasa: reporte de caso y revisión de la literatura}

- Humberto Martínez Cordero, Leonardo Enciso Olivera, Bonell Patiño Escobar, Diana Otero de la Hoz, Paola Spirko Sánchez, Sandra Isabel Chinchilla, Carmen Lucía Roa, Bibiana Martínez, Cindy Arévalo, Andrea Naranjo

Correo electrónico: humbertomartinez48@hotmail.com

Introducción: la crisis blástica $(C B)$ en la leucemia mieloide crónica (LMC) comprende en la mayoría de los casos el fenotipo mieloide o linfoide. La crisis blástica mielomastocítica es rara y el pronóstico es desconocido.

Métodos: descripción de un caso de crisis blástica mielomastocítica atendido en el Instituto Nacional de Cancerología.

Resultados: hombre de 29 años con diagnóstico de LMC desde el año 2010. Inicia tratamiento con imatinib sin alcanzar respuesta citogenética. Recibe rescate con dasatinib seguido de nilotinib, después de lo cual desarrolla una CB con un $72 \%$ de blastos, siendo llamativa la infiltración concomitante de la piel, lesiones óseas de tipo lítico e hipercalcemia que requirieron el uso de ácido zoledrónico como emergencia oncológica.
Luego de quimioterapia $7+3$ con bosutinib, documentamos un porcentaje de blastos del $29 \%$ en médula ósea y la existencia de un $46 \%$ de células con características mastocíticas. Se diagnostica crisis blástica mielomastocítica con diagnóstico diferencial de crisis basófila o mastocitosis sistémica agresiva con un trastorno hematológico clonal no hematológico. Se solicitaron niveles de triptasa sérica y mutación D816V C-KIT, que no fueron informados. El paciente muere por hiperleucocitosis y trombocitopenia severa con sangrado del sistema nervioso central.

Conclusión: no existen pautas en este escenario clínico debido a la rareza de esta entidad. Se asume que los pacientes mueren rápidamente después del diagnóstico. Son necesarios los registros de presentaciones inusuales de CB para definir estrategias de tratamiento. 